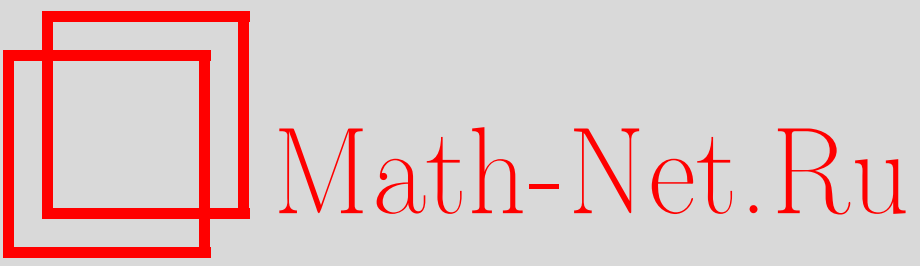

А. А. Битюрин, В. К. Манжосов, Математическое моделирование продольного удара системы однородных стержней о жёсткую преграду при неудерживающих связях, Вестн. Сам. гос. техн. ун-та. Сер. Физ.-мат. науки, 2009, выпуск 2(), 133-139

DOI: https://doi.org/10.14498/vsgtu652

Использование Общероссийского математического портала Math-Net.Ru подразумевает, что вы прочитали и согласны с пользовательским соглашением

http://www.mathnet.ru/rus/agreement

Параметры загрузки:

IP : 54.224 .187 .69

26 апреля 2023 г., 17:00:24 
УДК 517.958:622.233.6

\section{МАТЕМАТИЧЕСКОЕ МОДЕЛИРОВАНИЕ ПРОДОЛЬНОГО УДАРА СИСТЕМЫ ОДНОРОДНЫХ СТЕРЖНЕЙ О ЖЁСТКУЮ ПРЕГРАДУ ПРИ НЕУДЕРЖИВАЮЩИХ СВЯЗЯХ}

\section{А. А. Битюрин, В. К. Манжосов}

Ульяновский государственный технический университет, 432027, г. Ульяновск, ул. Северный Венец, 32.

E-mail: Denjgy0706@yandex.ru

Осуществляется математическое моделирование продолъного упругого иентралъного удара стержневой системы, состоящей из двух однородных стержней различной длины и площади поперечного сечения, о жёсткую преграду при неудерживающих связях.

Ключевые слова: деформачия, моделирование, продольный удар, стержень.

Введение. Задача о продольном ударе стержня с учётом его распределенной массы и описания движения поперечных сечений волновыми уравнениями была сформулирована Навье, Буссинеском, Сен-Венаном, Сирсом.

Во второй половине XX века применение ударных технологий в машиностроении, горнодобывающей промышленности, строительстве, приборостроении привело к значительному количеству теоретических и экспериментальных исследований в области продольного удара.

В известных работах модель учёта неудерживающих связей в задачах продольного удара стержней сводится к тому, что процесс удара считался завершенным, если в ударном сечении возникла деформация растяжения и происходил разрыв связи. Возможность повторного соударения стержней исследователями не рассматривалась. Такая модель продольного удара, с одной стороны, отсекала информацию о последующем нагружении стержня при повторных соударениях, а с другой стороны, представляла некорректную информацию о восстановлении скорости стержня при продольном ударе.

В данной работе представлена модель продольного удара стержней при разрывах связей и с учётом возникновения повторных соударений [1].

1. Постановка задачи. Рассматривается продольный удар однородного стержня 1, движущегося с предударной скоростью $V_{0}$, по стержню 2, взаимодействующему с жёсткой преградой. Длина первого стержня $l_{1}$ и масса $m_{1}$, длина и масса второго соответственно $l_{2}$ и $m_{2}$ (см. рис. 1). Общая длина обоих стержней $l=l_{1}+l_{2}$. Оба стержня состоят из одного материала. Используется волновая модель [1-5].

Движение поперечных сечений соударяемых стержней описывается волновыми уравнениями

$$
\frac{\partial^{2} u_{1}(x, t)}{\partial x^{2}}-\frac{1}{a^{2}} \frac{\partial^{2} u_{1}(x, t)}{\partial t^{2}}=0, \quad 0 \leqslant x \leqslant l_{1},
$$

Анатолий Александрович Битюрин (к.т.н.), старший преподаватель, каф. теоретической и прикладной механики. Владимир Кузъмич Манжосов (д.т.н., проф.), зав. кафедрой, каф. теоретической и прикладной механики. 


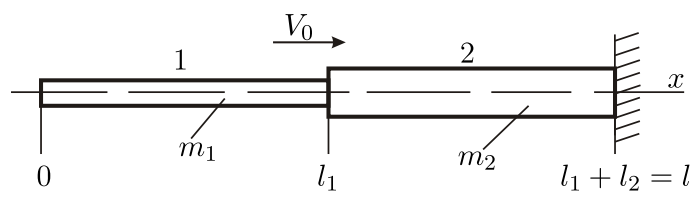

Рис. 1. Схема соударения однородных стержней

$$
\frac{\partial^{2} u_{2}(x, t)}{\partial x^{2}}-\frac{1}{a^{2}} \frac{\partial^{2} u_{2}(x, t)}{\partial t^{2}}=0, \quad l_{1} \leqslant x \leqslant l .
$$

Начальные условия определяют состояние стержней перед их соударением при $t=t_{0}=0$ :

$$
\frac{\partial u_{1}\left(x, t_{0}\right)}{\partial t}=V_{0}, \quad \frac{\partial u_{1}\left(x, t_{0}\right)}{\partial x}=0, \quad \frac{\partial u_{2}\left(x, t_{0}\right)}{\partial t}=0, \quad \frac{\partial u_{2}\left(x, t_{0}\right)}{\partial x}=0 .
$$

Краевые условия определяют отсутствие силы в сечении $x=0$ и равенство нулю скорости сечения $x=l$ при взаимодействии стержня 2 с жёсткой преградой:

$$
\frac{\partial u_{1}(0, t)}{\partial x}=0, \quad \frac{\partial u_{2}(l, t)}{\partial t}=0,
$$

а также равенство сил и условия сопряжения стержней в сечениях $x=l_{1}$ при непосредственном их взаимодействии:

$$
\begin{aligned}
E A_{1} \frac{\partial u_{1}\left(l_{1}, t\right)}{\partial x} & =E A_{2} \frac{\partial u_{2}\left(l_{1}, t\right)}{\partial x}, \quad \text { если } \quad \frac{\partial u_{1}\left(l_{1}, t\right)}{\partial x}<0, \\
\frac{\partial u_{1}\left(l_{1}, t\right)}{\partial t} & =\frac{\partial u_{2}\left(l_{1}, t\right)}{\partial t}, \quad \text { если } \quad \frac{\partial u_{1}\left(l_{1}, t\right)}{\partial x}<0,
\end{aligned}
$$

либо отсутствие сил в ударных сечениях стержней, если их взаимодействие отсутствует:

$$
\frac{\partial u_{1}\left(l_{1}, t\right)}{\partial x}=0, \quad \frac{\partial u_{2}\left(l_{1}, t\right)}{\partial x}=0, \quad \text { если } \quad u_{1}\left(l_{1}, t\right)-u_{2}\left(l_{1}, t\right) \leqslant 0,
$$

где $E$ - модуль упругости первого рода, $A_{1}$ и $A_{2}$ - площади поперечных сечений соответственно первого и второго стержней, $a$-скорость распространения продольной волны деформации.

2. Метод решения. Решение дифференциальных уравнений (1), (2) реализуется методом Даламбера [2] в следующем виде:

$$
\begin{gathered}
u_{1}(x, t)=f_{1}(a t-x)+\varphi_{1}(a t+x), \quad 0 \leqslant x \leqslant l_{1}, \\
u_{2}(x, t)=f_{2}(a t-x)+\varphi_{2}(a t+x), \quad l_{1} \leqslant x \leqslant l_{1}+l_{2}, \\
\varepsilon_{1}(x, t)=\frac{\partial u_{1}(x, t)}{\partial x}=-f_{1}^{\prime}(a t-x)+\varphi_{1}^{\prime}(a t+x), \\
v_{1}(x, t)=\frac{\partial u_{1}(x, t)}{\partial t}=a\left[f_{1}^{\prime}(a t-x)+\varphi_{1}^{\prime}(a t+x)\right], \\
\varepsilon_{2}(x, t)=\frac{\partial u_{2}(x, t)}{\partial x}=-f_{2}^{\prime}(a t-x)+\varphi_{2}^{\prime}(a t+x), \\
v_{2}(x, t)=\frac{\partial u_{2}(x, t)}{\partial t}=a\left[f_{2}^{\prime}(a t-x)+\varphi_{2}^{\prime}(a t+x)\right] .
\end{gathered}
$$


Перейдём к относительным величинам, характеризующим прямые и обратные волны:

$$
\tilde{f}^{\prime}(a t-x)=f^{\prime}(a t-x) /\left(\frac{V_{0}}{a}\right), \quad \tilde{\varphi}^{\prime}(a t+x)=\varphi^{\prime}(a t+x) /\left(\frac{V_{0}}{a}\right),
$$

а также деформацию в сечении и его скорость:

$$
\tilde{\varepsilon}(x, t)=-\tilde{f}^{\prime}(a t-x)+\tilde{\varphi^{\prime}}(a t+x), \quad \tilde{v}(x, t)=\frac{v(x, t)}{V_{0}}=\tilde{f}^{\prime}(a t-x)+\tilde{\varphi^{\prime}}(a t+x) .
$$

Относительные величины будут использоваться в дальнейшем.

Рассмотрим некоторое произвольное сечение $j$ на $i$-том интервале времени (см. рис. 2). Это сечение является границей сопряжения $j$-того и $j+1$-го участков. На сечение $j$ слева падает прямая волна $f_{j}\left(a t-x_{j-1}\right)$, сформированная на $(i-1)$-м интервале времени в $(j-1)$-м сечении, а справа - обратная волна $\varphi_{j+1}\left(a t+x_{j+1}\right)$, сформированная на $(i-1)$-м интервале времени в $(j+1)$-м сечении. Поскольку ударные волны в однородных участках имеют прямоугольный вид, волна $f_{j}\left(a t-x_{j-1}\right)$ иллюстрирована в виде левого верхнего прямоугольника переменного тона, а волна $\varphi_{j+1}\left(a t+x_{j+1}\right)-$ в виде правого верхнего прямоугольника.

Стрелками и направлением затемнённого растра указано направление распространения соответствующей волны деформации.

Интервал времени $\Delta t=\frac{\Delta l}{a}$ равен времени распространения волны деформации на участке длиной $\Delta l ; x_{j-1}$ и $x_{j+1}$ - координаты $j-1$-го и $j+1$-го сечений.

При преобразовании падающих волн в сечении $j$ формируется прямая волна $f_{j+1}\left(a t-x_{j}\right)$, распространяющаяся от сечения $j$ к сечению $j+1$, и обратная волна $\varphi_{j}\left(a t+x_{j}\right)$, распространяющаяся от сечения $j$ к сечению $j-1$. Причём производные функций определяются как [2]

$$
\begin{gathered}
f_{j+1}^{\prime}\left(a t-x_{j}\right)=q_{f}(j) f_{j}^{\prime}\left(a t-x_{j}\right)+r_{\varphi}(j) \varphi_{j+1}^{\prime}\left(a t+x_{j}\right), \\
\varphi_{j}^{\prime}\left(a t+x_{j}\right)=q_{\varphi}(j) \varphi_{j+1}^{\prime}\left(a t+x_{j}\right)+r_{f}(j) f_{j}^{\prime}\left(a t-x_{j}\right),
\end{gathered}
$$

где $q_{f}(j)=\frac{2}{\left(r_{j}+1\right) / r_{j}}-$ коэффициент прохождения прямой волны $f_{j}^{\prime}\left(a t-x_{j}\right)$, падающей на границу $x=x_{j}$ со стороны $j$-того участка; $r_{j}=\frac{A_{j}}{A_{j+1}}-$ отношение площадей поперечных сечений сопряженных $j$-того и $j+1$-го участков;

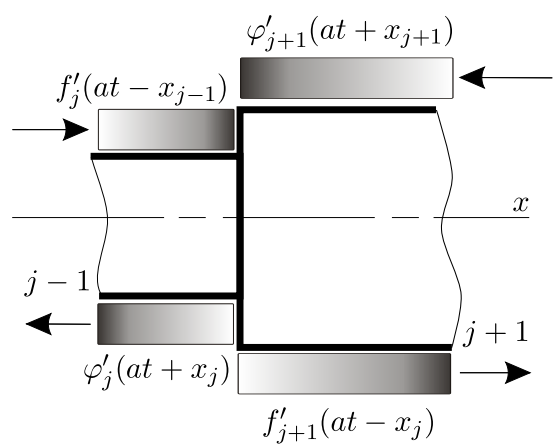

Рис. 2. Граница сопряжения однородных участков 
$r_{\varphi}(j)=\frac{1-r_{j}}{1+r_{j}}-$ коэффициент отражения обратной волны $\varphi_{j+1}^{\prime}\left(a t+x_{j}\right)$, падающей на границу $x=x_{j}$ со стороны $j+1$-го участка; $q_{\varphi}(j)=\frac{2}{r_{j}+1}-$ коэффициент прохождения обратной волны $\varphi_{j+1}^{\prime}\left(a t+x_{j}\right)$, падающей на границу $x=x_{j}$ со стороны $j-1$-го участка; $r_{f}(j)=\frac{r_{j}^{-} 1}{r_{j}+1}-$ коэффициент отражения прямой волны $f_{j}^{\prime}\left(a t-x_{j}\right)$, падающей на границу $x=x_{j}$ со стороны $j-1$-го участка.

Деформация в сечении $x_{j}$, принадлежащим $j$-тому участку, определится как

$$
\begin{aligned}
\varepsilon_{j}\left(x_{j}, t\right)=-f_{j}^{\prime}\left(a t+x_{j}\right)+q_{\varphi}(j) \varphi_{j+1}^{\prime}\left(a t+x_{j}\right)+r_{f}(j) f_{j}^{\prime}\left(a t-x_{j}\right)= \\
=-\left(1-r_{f}(j)\right) f_{j}^{\prime}\left(a t+x_{j}\right)+q_{\varphi}(j) \varphi_{j+1}^{\prime}\left(a t+x_{j}\right) .
\end{aligned}
$$

Деформация в сечении $x_{j}$, принадлежащим $j+1$-му участку, определится как:

$$
\begin{aligned}
\varepsilon_{j+1}\left(x_{j}, t\right)=-q_{f}(j) f_{j}^{\prime}(a t+ & \left.x_{j}\right)-r_{\varphi}(j) \varphi_{j+1}^{\prime}\left(a t+x_{j}\right)+\varphi_{j}^{\prime}\left(a t-x_{j}\right)= \\
& =-q_{f}(j) f_{j}^{\prime}\left(a t+x_{j}\right)+\left(1-r_{\varphi}(j)\right) \varphi_{j+1}^{\prime}\left(a t+x_{j}\right) .
\end{aligned}
$$

Равенство $\varepsilon_{j}\left(x_{j}, t\right)=\varepsilon_{j+1}\left(x_{j}, t\right)$ может быть только в том случае, когда $r_{f}(j)=0, r_{\varphi}(j)=0, q_{f}(j)=1, q_{\varphi}(j)=1$, а это возможно только тогда, когда $r_{j}=\frac{A_{j}}{A_{j+1}}=1$, т. е. при сопряжении однородных участков. Скорости сопряженных сечений участков всегда равны между собой $v_{j}\left(x_{j}, t\right)=v_{j+1}\left(x_{j}, t\right)$. Разница скоростей сопряженных сечений привела бы к разрыву стержня в этих сечениях.

3. Пример расчёта. Рассмотрим продольный упругий удар о жёсткую преграду однородных стержней с длинами $l_{1}=l_{2}=0,5 l$. Соотношение площадей поперечных сечений первого и второго стержней $\tilde{A}=\frac{A_{1}}{A_{2}}=0,5$ (см. рис. 2 ). Применим метод характеристик для построения поля состояний (см. рис. 3 ). Области состояний $\mathrm{I}_{0}-\mathrm{I}_{5}, \mathrm{II}_{0}-\mathrm{II}_{6}$ с соответствующими значениями $\tilde{f}^{\prime}(a t-x)$, $\tilde{\varphi}^{\prime}(a t+x), \tilde{\varepsilon}(x, t), \tilde{v}(x, t)$ определяют параметры прямых и обратных волн деформаций, продольную деформацию и скорость поперечных сечений. Длительность состояния для произвольного сечения определяется разностью ординат $t$, которые имеют точки наклонных линий для этого сечения.

При $t=2 l / a$ происходит отрыв однородного стержня 2 от жёсткой преграды, при $t=2,5 l / a$ происходит отрыв в контактном сечении $x=0,5 l$.

4. Анализ результатов. Авторами осуществлялось математическое моделирование продольного удара однородных стержней с длинами $l_{1}=0,2 l, l_{1}=$ $=0,4 l, l_{1}=0,5 l, l_{1}=0,6 l, l_{1}=0,8 l$ при соотношении площадей поперечных сечений предыдущего участка к последующему $\tilde{A}=0,5$ и $\tilde{A}=0,33$, где $\tilde{A}=\frac{A_{1}}{A_{2}}$. Анализ результатов моделирования удобнее проиллюстрировать на графике зависимости максимальной относительной продольной деформации в опасном сечении $\tilde{\varepsilon}_{\text {max }}$ от длины начального участка $l_{1}$ и величины $\tilde{A}$ (см. рис. 4).

Выводы.

1. При ударе однородного стержня о стержень, взаимодействующий с преградой, при $l_{1}=0,4 l, \widetilde{A}=0,5$ наблюдается повторное соударение стержней 


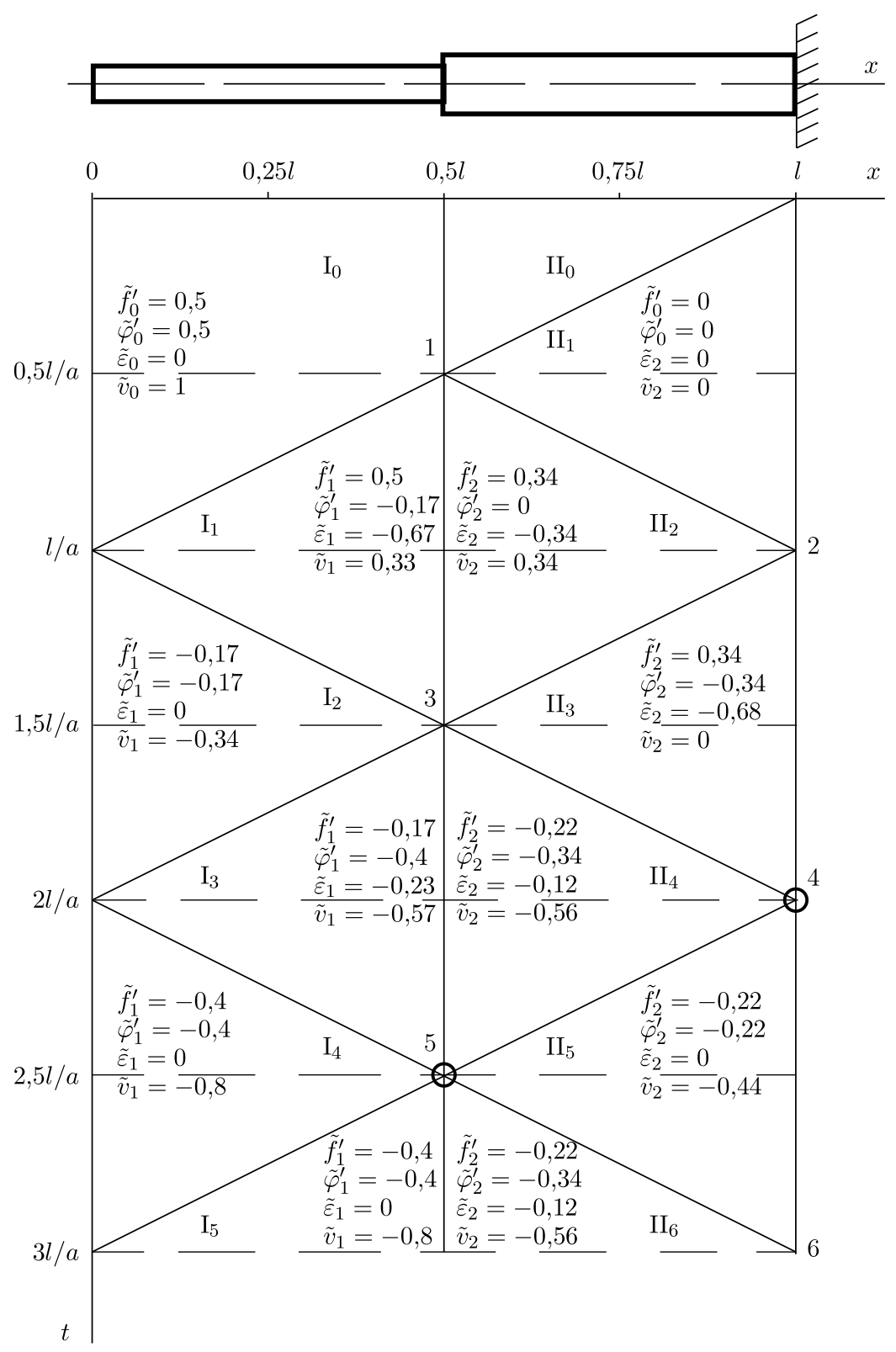

Рис. 3. Поле состояний при ударе системы однородных стержней о жёсткую преграду

в контактном сечении $x=l_{1}$. При других соотношениях длин однородных участков повторные соударения не наблюдаются.

2. Исходя из анализа графика следует отметить, что максимальная относительная продольная деформация $\tilde{\varepsilon}_{\max }$ в опасном сечении будет зависеть от длины $l_{1}$ и от параметра $\tilde{A}$. При переходе $l_{1}$ через значение $0,5 l$ максимальная деформация $\tilde{\varepsilon}_{\max }$ изменяется скачкообразно от значений $0,67 \div 0,75$ до $1,10 \div 1,12$ (рис. 4 ).

Используя данные математического моделирования, можно рассчитать значение абсолютной деформации (удлинение или укорочение) любого $i$-того 


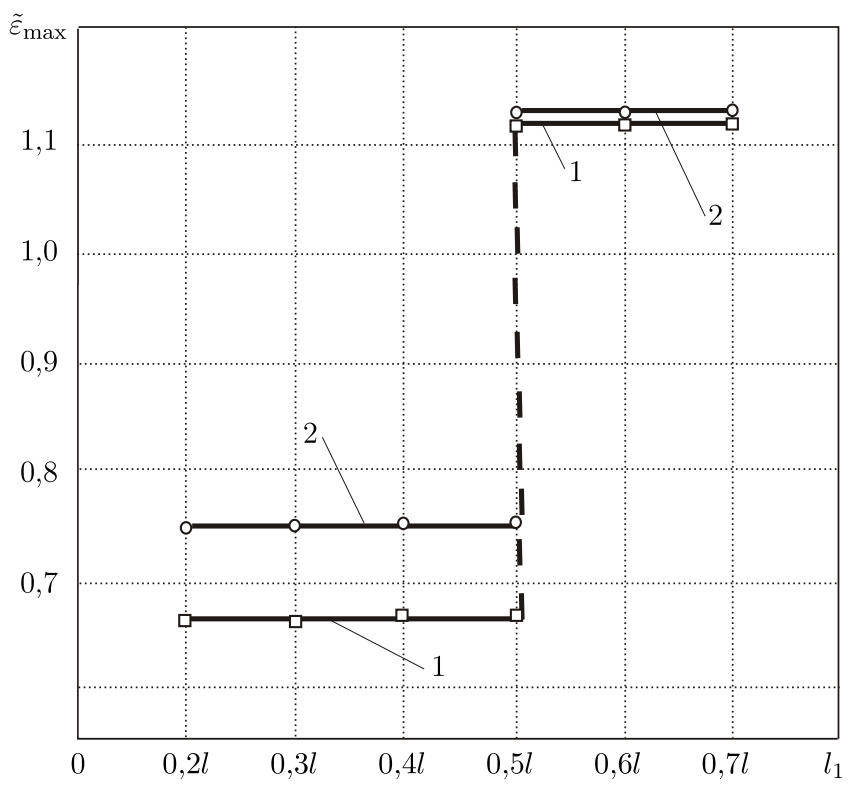

Рис. 4. Зависимость максимальной относительной продольной деформации $\tilde{\varepsilon}_{\max }: 1-$ $\tilde{\varepsilon}_{\max }\left(l_{1}\right)$ при $\tilde{A}=0,5 ; 2-\tilde{\varepsilon}_{\max }\left(l_{1}\right)$ при $\tilde{A}=0,33$

участка стержневой системы $\Delta l_{i}$, поскольку имеет место зависимость, полученная с учётом [6] и (3)-(8):

$$
\Delta l_{i}=l_{i} \tilde{\varepsilon} \frac{V_{0}}{a}
$$

где $\Delta l_{i}$ - абсолютная продольная деформация $i$-того участка стержневой системы, $l_{i}$ - длина этого участка.

Как видно, изменение линейных размеров стержневой системы будет зависеть от относительной продольной деформации, начальной предударной скорости и характеристик материала.

\section{БИБЛИОГРАФИЧЕСКИЙ СПИСОК}

1. Битюрин A.А., Манжсосов В.К. Изменение деформации на участках стержневой системы после повторного удара в контактном сечении // Вестн. УлГТУ, 2007. - № 3. C. $23-28$.

2. Александров E. В., Соколинский В. Б. Прикладная теория и расчёт ударных систем. М.: Наука, 1969. - 199 с.

3. Алимов О.Д., Манжосов В.К., Еремълни, В.Э. Распространение волн деформаций в ударных системах. - М.: Наука, 1985. - 354 с.

4. Битюрин A. А., Манжосов B. К. Моделирование продольного удара однородных стержней при неудерживающих связях // Вестн. УлГТУ, 2005. - № 3. - С. 23-25.

5. Манжосов В. К. Модели продольного удара. - Ульяновск, 2006. - 159 с.

6. Дарков А. В., Шпиро Г. С. Сопротивление материалов. - М.: Высш. шк., 2003. - 641 с. 
MSC: 65M25, 68T35, 12L12

MATHEMATICAL MODELING OF LONGITUDINAL BLOW OF THE SYSTEM OF HOMOGENEOUS RODS ABOUT RIGID BARRIER AT NOT-HOLDING CONNECTIONS

\section{A. A. Bityurin, V.K. Manzhosov}

Ulyanovsk State Technical University,

432027, Ulyanovsk, Nothern Venetz str., 32.

E-mail: Denjgy0706@yandex.ru

Mathematical modeling of the longitudinal elastic central blow of the rod system, consisting of two homogeneous rods of various lengths and the area of cross section over a rigid barrier is implemented at not-holding connections.

Key words: deformation, modeling, longitudinal blow, rod.

Original article submitted 04/XII/2008; revision submitted $15 / \mathrm{II} / 2009$.

Anatoliy A. Bityurin (Ph. D. (Techn.)), Lecturer, Dept. of Theoretical \& Applied Mechanics. Vladimir K. Manzhosov (Dr. Sci. (Techn.)), Head of Dept., Dept. of Theoretical \& Applied Mechanics. 